\title{
Singularity Analysis and Representation of 6DOF Parallel Robot Using Natural Coordinates
}

\author{
Shangyuan Zou $\mathbb{D}^{1},{ }^{1}$ Hairui Liu $\mathbb{D}^{2},{ }^{2}$ Yanli Liu $\mathbb{D}^{2,3}$ Jiafeng Yao $\mathbb{D}^{3},{ }^{3}$ and Hongtao Wu $\mathbb{D}^{3}$ \\ ${ }^{1}$ College of Transportation and Safty, Jiangsu College of Safety Technology, Xuzhou 221011, China \\ ${ }^{2}$ College of Electrical Engineering, Jiangsu College of Safety Technology, Xuzhou 221011, China \\ ${ }^{3}$ College of Mechanical and Electrical Engineering, Nanjing University of Aeronautics \& Astronautics, Nanjing 210016, China
}

Correspondence should be addressed to Shangyuan Zou; mezousy@126.com

Received 4 March 2021; Accepted 24 May 2021; Published 10 June 2021

Academic Editor: Ruthber Rodriguez Serrezuela

Copyright (c) 2021 Shangyuan Zou et al. This is an open access article distributed under the Creative Commons Attribution License, which permits unrestricted use, distribution, and reproduction in any medium, provided the original work is properly cited.

\begin{abstract}
Singularity research is carried out. The problem, which is about six-dimensional parameters of position and orientation can not realize three-dimensional visualization for 6DOF parallel robot, has been solved. Firstly, according to the structural characteristics of the 6DOF parallel robot with the planar platform, the position and orientation of the mobile platform are described, respectively, and the six equations of forward kinematics are established by choosing the natural coordinates of three representative points as parameters. Then, the singularities of the $6 \mathrm{DOF}$ parallel robot with a planar platform are divided into input singularity and output singularity. Aiming at the output singularity, in combination with six constraint equations among the position vectors of three representative points, an analytical algorithm is proposed to express the coupling singularity of position and orientation and the analytical expression is derived. In further research, three kinds of output singularities are found, the spatial distribution of the output singular trajectory is determined, and a unified three-dimensional fully visualized description of six-dimensional coupling variables is realized for the first time. The problems of finding the singular orientation at a given position or the singular position at a given orientation are solved. The analysis of the singularity lays a solid foundation for the description of the threedimensional complete visualization of a six-dimensional singularity-free workspace based on forward kinematics. What is more, it has great significance for both trajectory planning and control design of the parallel robot.
\end{abstract}

\section{Introduction}

In recent years, $6 \mathrm{DOF}$ parallel robots are more and more widely used in VR, entertainment, medical and aerospace simulators, wave compensation simulators, radio telescopes (FAST), and so on. The requirements of high speed, high precision, high rigidity, high dynamic performance, low inertia, small structure size, and other performance are also higher and higher. If the parallel robot meets these performance requirements, it is necessary to avoid singular configuration.

When the mechanism is in a special configuration, the normal degree of freedom changes instantaneously, which means the mechanism exits singularity. There are many methods to study the singularity of the 6DOF parallel robot.
The first method to study the singularity of the 6DOF parallel robot is based on the screw theory. In the 1970s, Hunt [1] first used the screw theory to analyze the singularity of the parallel mechanism. Yan Wen et al. [2] proposed a singular kinematic theory by using the screw theory. Cao et al. [3] also used the screw theory to study the singularity. Laryushkin et al. [4] used the screw theory to study the singularity of the 3DOF translational parallel mechanism and a planar parallel mechanism. The problem of singularity is an important mechanical characteristic of the parallel robot, which is one of the research hotspots for the parallel robot. What is more, the study of singularity is very crucial for the design and control of the parallel robot. Gosselin and Angeles [5] first proposed an analysis method based on the input-output speed and divided the singularities into 
boundary singularity, configuration singularity, and structure singularity, in which the configuration singularity is the main problem that we studied.

When the mechanism is singular, the corresponding configuration is singular. Singular configuration is an important basis for the determination of performance indexes such as nonsingular workspace, flexibility, and isotropy. Singular configuration includes input and output singularities, which need to be considered and avoided in solving the maximum nonsingular workspace $[6,7]$, trajectory planning, control, and other stages. In order to better understand the nature of mechanism singular configuration and better avoid the singular configuration and its surrounding areas in practical application, many scholars at home and abroad have launched the research on singular configuration.

The singular configuration was first discovered by Hunt [8]. The classical methods to study singular configuration are the Grassmann line geometry method and the Jacobian matrix method. Merlet [9] introduced the Grassmann line geometry method to analyze the singularity of 6-3 platform and established the geometric conditions of singular configuration. Wen et al. [10] studied the singularities of 3-DOF planar parallel manipulators using Grassmann-Cayley algebra. Ma et al. [11] studied 3/6-SPS Stewart manipulator by using the method of Grassmann-Cayley algebra. The positionsingularity loci and orientation-singularity loci are drawn based on the polynomial obtained from the coefficient of the outer product of all 6 line vectors. Although it is convenient and intuitive to verify the singularities by the method of Grassmann linear geometry, it is difficult to find the singularities of the whole distribution. Scholars such as Choi et al. [12] and Choi and Ryu [13] used the Jacobian matrix method to study the singular configuration of the 4-DOF parallel robot and 4-DOF parallel manipulator, respectively.

A proper model is established by selecting the natural coordinates formed by the appropriate points and orientation vectors to describe the multibody system [14]. And the $13^{\text {th }}$ order analytical polynomial of singular trajectory for a kind of parallel mechanism is derived using the half-angle conversion method [3].

At present, Li et al. [15] were all given orientation parameters to find position singularity. Cao et al. [3] had also studied how to find the orientation singularity at the given position parameters. In addition, singular trajectories $[16,17]$ are represented by three-dimensional parameters in three-dimensional space. Although position and orientation parameters are coupled, the complete visualization of position and orientation singular trajectories is independent of each other, which does not realize the complete visualization of the six parameters in three-dimensional space. Therefore, the study of position and orientation singularities greatly affects the complete visualization of position and orientation coupling parameters. What is more, it leads to the solution and representation of the largest nonsingular workspace.

In conclusion, in order to make the six-dimensional parameters of position and orientation be fully visualized in the three-dimensional space, the natural coordinates method is used to represent the position and orientation and six kinematic equations are deduced in this paper. In order to divide the traditional research of singularity into input singularity and output singularity, the kinematic equations are divided into two parts and combined with two different sets of constraint equations. Aiming at the output singularity, the analytical expressions of output pose singular trajectory are obtained, and the types of output singularity are analyzed. What is more, the complete visualization of six-dimensional position and orientation parameter singularity in three-dimensional space is realized for the first time. The study of output singularities lays a solid foundation for solving the maximum nonsingular workspace, trajectory planning [18], and control [19].

\section{Kinematics Foundation of 6DOF Parallel Robot}

2.1. Structure. The $6 \mathrm{DOF}$ parallel robot with the planar platform and its coordinate system is shown in Figure 1. The mechanism consists of two platforms, the base and the mobile platform, and six legs with identical structures. $A_{i}$ and $B_{i}$ are the centers of spherical joint $S$ and universal joint $U$, respectively. All $A_{i}$ and $B_{i}$ are restricted to a plane, respectively; that is, this $6 \mathrm{DOF}$ parallel robot belongs to the planar type. In order to facilitate the analysis, $O_{b} x y z$, the absolute static frame, is selected to be fixedly connected with the base, and $O_{a} \alpha \beta \gamma$, the relative moving frame, is fixedly connected with the mobile platform, respectively. $O_{a}$ and $O_{b}$ are the centers of the mobile platform and the base, respectively. The $z$ and $\gamma$ axes are perpendicular to their respective planes, respectively.

Three points, $P_{1}, P_{2}, P_{3}$, are selected as natural coordinates, which are located at the coordinate origin, $\alpha$ axis endpoint and $\beta$ axis endpoint of the moving frame $O_{a} \alpha \beta \gamma$, respectively, as shown in Figure 1. The vectors, $P_{1}=\left\{x_{1}\right.$, $\left.y_{1}, z_{1}\right\}^{T}, P_{2}=\left\{x_{2}, y_{2}, z_{2}\right\}^{T}$, and $P_{3}=\left\{x_{3}, y_{3}, z_{3}\right\}^{T}$, are used to represent three position coordinates of three natural coordinates, $P_{1}, P_{2}$, and $P_{3}$, in the static frame. As a result, nine variables to be solved are included in the forward kinematics model.

2.2. Represent Position $P$ and Orientation $R$ with Natural Coordinates. There exists the following relationship among $P_{2}, P_{3}$, and $P_{1}$, as follows: $P_{2}=P_{1}+R \cdot x, P_{3}=P_{1}+R \cdot y$. The vector coordinates of $\alpha$ axis, $\beta$ axis, and $\gamma$ axis in the moving frame $O_{a} \alpha \beta \gamma$ are remembered as $\alpha=\left\{\alpha_{x}, \alpha_{y}, \alpha_{z}\right\}^{T}$, $\beta=\left\{\beta_{x}, \beta_{y}, \beta_{z}\right\}^{T}$, and $\gamma=\left\{\gamma_{x}, \gamma_{y}, \gamma_{z}\right\}^{T}$. Then, $\alpha, \beta, \gamma$ are represented with natural coordinates of the three representative points $P_{1}, P_{2}, P_{3}$, as follows:

$$
\begin{aligned}
\alpha & =P_{2}-P_{1}=\left\{x_{2}, y_{2}, z_{2}\right\}^{T}-\left\{x_{1}, y_{1}, z_{1}\right\}^{T} \\
& =\left\{\left(x_{2}-x_{1}\right),\left(y_{2}-y_{1}\right),\left(z_{2}-z_{1}\right)\right\}^{T}
\end{aligned}
$$

Similarly, we obtain

$$
\beta=\left\{\left(x_{3}-x_{1}\right),\left(y_{3}-y_{1}\right),\left(z_{3}-z_{1}\right)\right\}^{T} \text {. }
$$

Because $\gamma$ is perpendicular to the plane determined by $\alpha$ and $\beta$, there is 


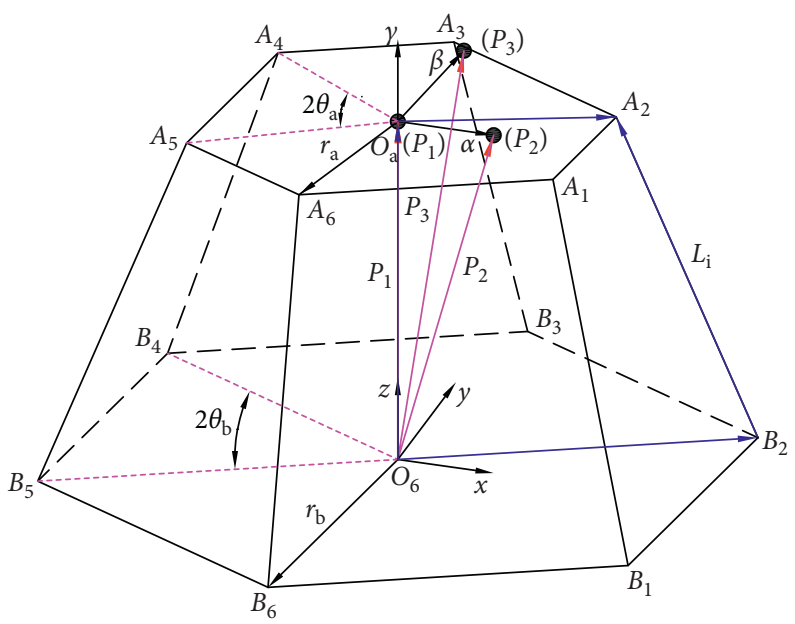

FIGURE 1: Sketch of the 6DOF parallel robot with the planar platform. There are two platforms and six legs. $A_{i}$ are the vertices of the moving platform. $B i$ are the vertices of the base. $O_{a} \alpha \beta \gamma$ is the relative moving frame. $O_{b} x y z$ is the absolute static frame. $\mathrm{P}_{1}, \mathrm{P}_{2}$, and $\mathrm{P}_{3}$ are selected as natural coordinates. $O_{a}$ and $O_{b}$ are the circle center of the two platforms. $r_{a}, r_{b}$ are circumradii of the base and the moving platform, respectively. $\theta_{a}, \theta_{b}$ are center semiangles corresponding to the short side of the platform, respectively.

$$
\begin{aligned}
\gamma= & \alpha \times \beta=\left\{\left(\alpha_{y} \beta_{z}-\alpha_{z} \beta_{y}\right),\left(\alpha_{z} \beta_{x}-\alpha_{x} \beta_{z}\right),\left(\alpha_{x} \beta_{y}-\alpha_{y} \beta_{x}\right)\right\}^{T} \\
= & \left\{\left(y_{1} z_{2}-y_{1} z_{3}-y_{2} z_{1}+y_{2} z_{3}+y_{3} z_{1}-y_{3} z_{2}\right),\left(-x_{1} z_{2}+x_{1} z_{3}+x_{2} z_{1}-x_{2} z_{3}-x_{3} z_{1}+x_{3} z_{2}\right),\right. \\
& \left.\left(x_{1} y_{2}-x_{1} y_{3}-x_{2} y_{1}+x_{2} y_{3}+x_{3} y_{1}-x_{3} y_{2}\right)\right\}^{T} .
\end{aligned}
$$

The base vector of the static coordinate system is $x=\{1,0,0\}^{T}, \quad y=\{0,1,0\}^{T}, \quad z=\{0,0,1\}^{T}$. The position vector, represented by natural coordinates, of the moving platform in the static coordinate system is $P=P_{1}=\left\{x_{1}, y_{1}, z_{1}\right\}^{T}$; the orientation matrix $R$ is expressed as

$$
R=[\alpha, \beta, \gamma]=\left[\begin{array}{ccc}
\alpha_{x} & \beta_{x} & \gamma_{x} \\
\alpha_{y} & \beta_{y} & \gamma_{y} \\
\alpha_{z} & \beta_{z} & \gamma_{z}
\end{array}\right]
$$

where each component in equation (4) is obtained according to equations (1)-(3), as follows:

$$
\begin{aligned}
& \alpha_{x}=x_{2}-x_{1}, \\
& \alpha_{y}=y_{2}-y_{1}, \\
& \alpha_{z}=z_{2}-z_{1}, \\
& \beta_{x}=x_{3}-x_{1}, \\
& \beta_{y}=y_{3}-y_{1}, \\
& \beta_{z}=z_{3}-z_{1}, \\
& \gamma_{x}=y_{1} z_{2}-y_{1} z_{3}-y_{2} z_{1}+y_{2} z_{3}+y_{3} z_{1}-y_{3} z_{2}, \\
& \gamma_{y}=-x_{1} z_{2}+x_{1} z_{3}+x_{2} z_{1}-x_{2} z_{3}-x_{3} z_{1}+x_{3} z_{2}, \\
& \gamma_{z}=x_{1} y_{2}-x_{1} y_{3}-x_{2} y_{1}+x_{2} y_{3}+x_{3} y_{1}-x_{3} y_{2} .
\end{aligned}
$$

\section{Kinematics Model of 6DOF Parallel Robot}

3.1. Construction Vector Equation and Separation Primary and Secondary Variables. As shown in Figure 1, there are 6 groups of closed vectors in the mechanism, namely, $\mathrm{O}_{b} \vec{O}_{a} A_{1}$ $\mathrm{O}_{b} \vec{B}_{1} A_{1}, \quad \mathrm{O}_{b} \vec{O}_{a} A_{2} \quad \mathrm{O}_{b} \vec{B}_{2} A_{2}, \quad \ldots, \quad \mathrm{O}_{b} \vec{O}_{a} A_{6} \quad O_{b} \vec{B}_{6} A_{6}$. According to the vector closed relationship, the six extendable link vectors of six groups of closed vectors are expressed as

$$
L_{k} e_{k}=P+R a_{k}-b_{k}, \quad(k=1, \ldots),
$$

where $L_{k}$ is the length of the $k$ th extendable link; $e_{k}$ is the unit vector of the $k$ th extendable link; $P$ is the position vector of the moving platform in static frame, namely, $P=P_{1}=\left\{x_{1}, y_{1}, z_{1}\right\}^{T} ; R$ is the orientation matrix, shown in equation (4); $a_{k}$ is the coordinates of vertices $A_{k}$ of the mobile platform in the moving frame $O_{a} \alpha \beta \gamma$, namely, $a_{k}=\left\{a_{k \alpha}, a_{k \beta}, a_{k \gamma}\right\}^{T}$; and $b_{k}$ is the coordinates of vertices $B_{k}$ of the base platform in the basic frame $O_{b} x y z$, namely, $b_{k}=\left\{b_{k x}, b_{k y}, b_{k z}\right\}^{T}$. As shown in Figure 1, because the vertices of the mobile platform and base platform are all arranged in a plane, the $\gamma$ axis and $z$ axis components of $a_{k}, b_{k}$ are all zero, namely, $a_{k \gamma}=b_{k z}=0$.Thus, $a_{k}, b_{k}$ are expressed as $a_{k}=\left\{a_{k \alpha}, a_{k \beta}, 0\right\}^{T}, b_{k}=\left\{b_{k x}, b_{k y}, 0\right\}^{T}$. It can be seen that the vertex coordinates of mobile and static platforms are also expressed by four variables $r_{a}, r_{b}, \theta_{a}, \theta_{b}$, and the coordinates of each point are further expressed as follows: 


$$
\begin{aligned}
& a_{k}=\left\{a_{k \alpha}, a_{k \beta}, a_{k \gamma}\right\}^{T}=\left\{r_{a} \cos \left(\varphi_{k}\right), r_{a} \sin \left(\varphi_{k}\right), 0\right\}^{T}, \\
& b_{k}=\left\{b_{k x}, b_{k y}, b_{k z}\right\}^{T}=\left\{r_{b} \cos \left(\beta_{k}\right), r_{b} \sin \left(\beta_{k}\right), 0\right\}^{T},
\end{aligned}
$$

where

$$
\begin{aligned}
& \varphi_{k}=\frac{(k-1) \pi}{3}-\theta_{a}, \beta_{k}=\frac{(k-1) \pi}{3}-\theta_{b}, \quad k=1,3,5, \\
& \varphi_{k}=\frac{(k-2) \pi}{3}+\theta_{a}, \beta_{k}=\frac{(k-2) \pi}{3}+\theta_{b}, \quad k=2,4,6 .
\end{aligned}
$$

Let $W$ be the position vector of the mobile platform in the moving frame, $W=\left\{W_{x}, W_{y}, W_{z}\right\}^{T}$; then, there exists $P=\mathrm{RW}$. In combination with the orthogonality of $R$, there exists $W=R^{T} P$. By substituting $a_{k}, b_{k}, P, R$, and $W$ into equation (6) and dot multiplication of the two sides of the equation with their own vector, the six links length square scalar equations are obtained as follows (the subscript $\mathrm{k}$ is omitted here):

$$
\begin{aligned}
L^{2}-r_{a}^{2}-r_{b}^{2}= & -2 b_{x} P_{x}-2 b_{y} P_{y}+2 a_{x} W_{x}+2 a_{y} W_{y}-2 \alpha_{x}\left(a_{x} b_{x}\right) \\
& -2 \alpha_{y}\left(a_{x} b_{y}\right)-2 \beta_{x}\left(a_{y} b_{x}\right)-2 \beta_{y}\left(a_{y} b_{y}\right)+P_{p} .
\end{aligned}
$$

In the previous equation, there are 9 unknowns, including $P_{p}, P_{x}, P_{y}, W_{x}, W_{y}, \alpha_{x}, \alpha_{y}, \beta_{x}$, and $\beta_{y}$. The 9 unknowns are related by the position and orientation parameters of the mobile platform. Furthermore, the coefficients of the 9 unknowns are determined by the structural parameters and the length parameters of the platform, where $P_{p}$ is the square of module length of position vector $P . P_{x}$ is the projection of $P$ in direction $x$, namely, $P_{x}=P . x$.
Similarity, $P_{y}=P . y, W_{x}=P . \alpha$, and $W_{y}=P . \beta . \alpha_{x}$ is the projection of $\alpha$ in direction $x$, namely, $\alpha_{x}=\alpha$.x. Similarity, $\alpha_{y}=\alpha . y, \beta_{x}=\beta . x$, and $\beta_{y}=\beta . y$.

3.2. Kinematic Model Represented by Natural Coordinates. Based on equation (9), the nine unknowns of $P_{p}, P_{x}, P_{y}, W_{x}$, $W_{y}, \alpha_{x}, \alpha_{y}, \beta_{x}$, and $\beta_{y}$ are transferred and sorted out; then, we can obtain the following results:

$$
\left\{\begin{array}{l}
P_{P}-\frac{k_{0}\left(\alpha_{x}+\beta_{y}\right)}{2}=C_{1} \\
P_{x}-\frac{k_{1}\left(\alpha_{x}-\beta_{y}\right)}{2}=C_{2} \\
P_{y}+\frac{k_{1}\left(\alpha_{y}+\beta_{x}\right)}{2}=C_{3} \\
W_{x}-\frac{k_{2}\left(\alpha_{x}-\beta_{y}\right)}{2}=C_{4} \\
W_{y}+\frac{k_{2}\left(\alpha_{y}+\beta_{x}\right)}{2}=C_{5} \\
\frac{\left(\alpha_{y}-\beta_{x}\right)}{2}=C_{6}
\end{array}\right.
$$

where

$$
\begin{aligned}
& k_{0}=2 r_{a} r_{b} \cos \left[\theta_{b}-\theta_{a}\right], k_{1}=r_{a} \sin \left[\theta_{b}+2 \theta_{a}\right] \operatorname{Csc}\left[\theta_{b}-\theta_{a}\right], k_{2}=r_{b} \sin \left[2 \theta_{b}+\theta_{a}\right]\left[\operatorname{Csc}\left[\theta_{b}-\theta_{a}\right]\right] \\
& C_{1}=\frac{1}{6}\left[L_{1}^{2}+L_{2}^{2}+L_{3}^{2}+L_{4}^{2}+L_{5}^{2}+L_{6}^{2}-6 r_{b}^{2}-6 r_{a}^{2}\right] \\
& C_{2}=\frac{-1}{12 r_{b}} \operatorname{Csc}\left[\theta_{b}-\theta_{a}\right]\left[\sqrt{3}\left(L_{3}^{2}-L_{4}^{2}-L_{5}^{2}+L_{6}^{2}\right) \cos \left[\theta_{a}\right]+\left(-2 L_{1}^{2}-2 L_{2}^{2}+L_{3}^{2}+L_{4}^{2}+L_{5}^{2}+L_{6}^{2}\right) \sin \left[\theta_{a}\right]\right] \\
& C_{3}=\frac{1}{12 r_{b}} \operatorname{Csc}\left[\theta_{b}-\theta_{a}\right]\left[\left(2 L_{1}^{2}-2 L_{2}^{2}-L_{3}^{2}+L_{4}^{2}-L_{5}^{2}+L_{6}^{2}\right) \cos \left[\theta_{a}\right]+\sqrt{3}\left(L_{3}^{2}+L_{4}^{2}-L_{5}^{2}-L_{6}^{2}\right) \sin \left[\theta_{a}\right]\right] \\
& C_{4}=\frac{-1}{12 r_{a}} \operatorname{Csc}\left[\theta_{b}-\theta_{a}\right]\left[\sqrt{3}\left(L_{3}^{2}-L_{4}^{2}-L_{5}^{2}+L_{6}^{2}\right) \cos \left[\theta_{b}\right]+\left(-2 L_{1}^{2}-2 L_{2}^{2}+L_{3}^{2}+L_{4}^{2}+L_{5}^{2}+L_{6}^{2}\right) \sin \left[\theta_{b}\right]\right] \\
& C_{5}=\frac{1}{12 r_{a}} \operatorname{Csc}\left[\theta_{b}-\theta_{a}\right]\left[\left(2 L_{1}^{2}-2 L_{2}^{2}-L_{3}^{2}+L_{4}^{2}-L_{5}^{2}+L 6^{2}\right) \cos \left[\theta_{b}\right]+\sqrt{3}\left(L_{3}^{2}+L_{4}^{2}-L_{5}^{2}-L_{6}^{2}\right) \operatorname{Sin}\left[\theta_{b}\right]\right] \\
& C_{6}=\frac{1}{12 r_{a} r_{b}} \operatorname{Csc}\left[\theta_{b}-\theta_{a}\right]\left[\left(L_{1}^{2}-L_{2}^{2}+L_{3}^{2}-L_{4}^{2}+L_{5}^{2}-L_{6}^{2}\right)\right] .
\end{aligned}
$$

By substituting the representative point expressions of 9 unknowns into equation (10), six polynomial equations with degree one or quadratic form can be obtained as follows: 


$$
\begin{array}{r}
x_{1}^{2}+y_{1}^{2}+z_{1}^{2}-\frac{1}{2} k_{0}\left(-x_{1}+x_{2}-y_{1}+y_{3}\right)=C_{1}, \\
x_{1}-\frac{1}{2} k_{1}\left(-x_{1}+x_{2}+y_{1}-y_{3}\right)=C_{2}, \\
y_{1}+\frac{1}{2} k_{1}\left(-x_{1}+x_{3}-y_{1}+y_{2}\right)=C_{3}, \\
x_{1}\left(-x_{1}+x_{2}\right)+y_{1}\left(-y_{1}+y_{2}\right)+z_{1}\left(-z_{1}+z_{2}\right)-\frac{1}{2} k_{2}\left(-x_{1}+x_{2}+y_{1}-y_{3}\right)=C_{4}, \\
x_{1}\left(-x_{1}+x_{3}\right)+y_{1}\left(-y_{1}+y_{3}\right)+z_{1}\left(-z_{1}+z_{3}\right)+\frac{1}{2} k_{2}\left(-x_{1}+x_{3}-y_{1}+y_{2}\right)=C_{5}, \\
\frac{1}{2}\left(x_{1}-x_{3}-y_{1}+y_{2}\right)=C_{6} .
\end{array}
$$

\section{Output Singularities and Analysis of 6DOF Parallel Robot}

In this paper, the output singularities of planar $6 \mathrm{DOF}$ parallel robot are studied. What is more, the types of output singularities are discussed.

4.1. Output Jacobian Matrix Represented by Natural Coordinates. By the relationships among the position vectors, $P_{1}, P_{2}, P_{3}$, represented by natural coordinates, remember $\tau=\left\{\tau_{1}, \tau_{2}, \tau_{3}\right\}$; that is,

$$
\begin{aligned}
& \tau_{1}=P_{1} \cdot P_{1}=x_{1}^{2}+y_{1}^{2}+z_{1}^{2}, \\
& \tau_{2}=P_{2} \cdot P_{2}=x_{2}^{2}+y_{2}^{2}+z_{2}^{2}, \\
& \tau_{3}=P_{3} \cdot P_{3}=x_{3}^{2}+y_{3}^{2}+z_{3}^{2} .
\end{aligned}
$$

From vector closed relationships in Figure 1, we can obtain

$$
\begin{aligned}
& P_{1}=P, \\
& P_{2}=P_{1}+\alpha=P+R \cdot x, \\
& P_{3}=P_{1}+\beta=P+R \cdot y .
\end{aligned}
$$

After substituting $x=\{1,0,0\}^{T}$ and $y=\{0,1,0\}^{T}$ into equation (14), $P_{1}, P_{2}$, and $P_{3}$ become compound variables including position and orientation parameters. Therefore, $\tau_{1}, \tau_{2}$, and $\tau_{3}$ are also compound variables with position and orientation parameters.

From equation (14), we obtain

$$
P_{2}-P_{1}=\alpha .
$$

Dot product with their own of two sides in equation (15). Combination with the unit vector $\alpha=\{1,0,0\}$, we obain,

$$
P_{2} \cdot P_{2}+P_{1} \cdot P_{1}-2 P_{1} \cdot P_{2}=1 \text {. }
$$

Therefore, there is

$$
P_{1} \cdot P_{2}=\frac{1}{2}\left(P_{1} \cdot P_{1}+P_{2} \cdot P_{2}-1\right)=\frac{1}{2}\left(\tau_{1}+\tau_{2}-1\right),
$$

and

$$
P_{1} \cdot P_{2}=\left\{x_{1}, y_{1}, z_{1}\right\}^{T} \cdot\left\{x_{2}, y_{2}, z_{2}\right\}^{T}=x_{1} x_{2}+y_{1} y_{2}+z_{1} z_{2} .
$$

Therefore, there is

$$
P_{1} \cdot P_{2}=\frac{1}{2}\left(\tau_{1}+\tau_{2}-1\right)=x_{1} x_{2}+y_{1} y_{2}+z_{1} z_{2} \text {. }
$$

Similarly, we obtain

$$
\begin{aligned}
& P_{1} \cdot P_{3}=\frac{1}{2}\left(\tau_{1}+\tau_{3}-1\right)=x_{1} x_{3}+y_{1} y_{3}+z_{1} z_{3}, \\
& P_{2} \cdot P_{3}=\frac{1}{2}\left(\tau_{2}+\tau_{3}-1\right)=x_{2} x_{3}+y_{2} y_{3}+z_{2} z_{3} .
\end{aligned}
$$

Therefore, we can obtain twelve equations which are made up of equations (12), (13), (19), (20), and (21).

First, equation (13) is substituted into equation (12). Then, the natural coordinates of representative points $P_{1}, P_{2}$ and $P_{3}$ are also substituted into a new equation (12):

$$
\begin{array}{r}
-C_{1}-\frac{1}{2} k_{0}\left(-x_{1}+x_{2}-y_{1}+y_{3}\right)+\tau_{1}=0 \\
-C_{2}+x_{1}-\frac{1}{2} k_{1}\left(-x_{1}+x_{2}+y_{1}-y_{3}\right)=0 \\
-C_{3}+y_{1}+\frac{1}{2} k_{1}\left(-x_{1}+x_{3}-y_{1}+y_{2}\right)=0 \\
-C_{4}-\frac{1}{2} k_{2}\left(-x_{1}+x_{2}+y_{1}-y_{3}\right)+\frac{1}{2}\left(-1-\tau_{1}+\tau_{2}\right)=0 \\
-C_{5}+\frac{1}{2} k_{2}\left(-x_{1}+x_{3}-y_{1}+y_{2}\right)+\frac{1}{2}\left(-1-\tau_{1}+\tau_{3}\right)=0 \\
-C_{6}+\frac{1}{2}\left(x_{1}-x_{3}-y_{1}+y_{2}\right)=0 .
\end{array}
$$

Equations (13), (19), (20), and (21) are resorted out as follows: 


$$
\begin{aligned}
x_{1}^{2}+y_{1}^{2}+z_{1}^{2}-\tau_{1} & =0, \\
x_{2}^{2}+y_{2}^{2}+z_{2}^{2}-\tau_{2} & =0, \\
x_{3}^{2}+y_{3}^{2}+z_{3}^{2}-\tau_{3} & =0, \\
x_{1} x_{2}+y_{1} y_{2}+z_{1} z_{2}+\frac{1}{2}\left(1-\tau_{1}-\tau_{2}\right) & =0, \\
x_{1} x_{3}+y_{1} y_{3}+z_{1} z_{3}+\frac{1}{2}\left(1-\tau_{1}-\tau_{3}\right) & =0, \\
x_{2} x_{3}+y_{2} y_{3}+z_{2} z_{3}+\frac{1}{2}\left(1-\tau_{2}-\tau_{3}\right) & =0
\end{aligned}
$$

Therefore, 12 new kinematic equations, represented with natural coordinates, are obtained by combination with equations (22) and (23).
By calculating the first partial derivative of time $t$ for 12 variables $x_{1}, y_{1}, z_{1}, x_{2}, y_{2}, z_{2}, x_{3}, y_{3}, z_{3}, \tau_{1}, \tau_{2}, \tau_{3}$ from the kinematic equations of equations (22) and (23), equations (22) and (23) are uniformly expressed as the vector forms as follows:

$$
J_{2} \cdot V_{x}=V_{c}
$$

where $V_{x}=\left\{\dot{x}_{1}, \dot{y}_{1}, \dot{z}_{1}, \dot{x}_{2}, \dot{y}_{2}, \dot{z}_{2}, \dot{x}_{3}, \dot{y}_{3}, \dot{z}_{3}, \dot{\tau}_{1}, \dot{\tau}_{2}, \dot{\tau}_{3}\right\}^{T}$ and $V_{c}$ is the velocities about six extendable links. The coefficient matrix is a linear transformation matrix between $V_{x}$ and $V_{c}$. Therefore, $J_{2}$ is the $12 \times 12$ dimensional Jacobian matrix of the mobile platform. It contains the natural coordinates of representative points in $J_{2}$, with dimensional consistency. So, it does not need to be dimensionless processed. The specific form of $J_{2}$ is as follows:

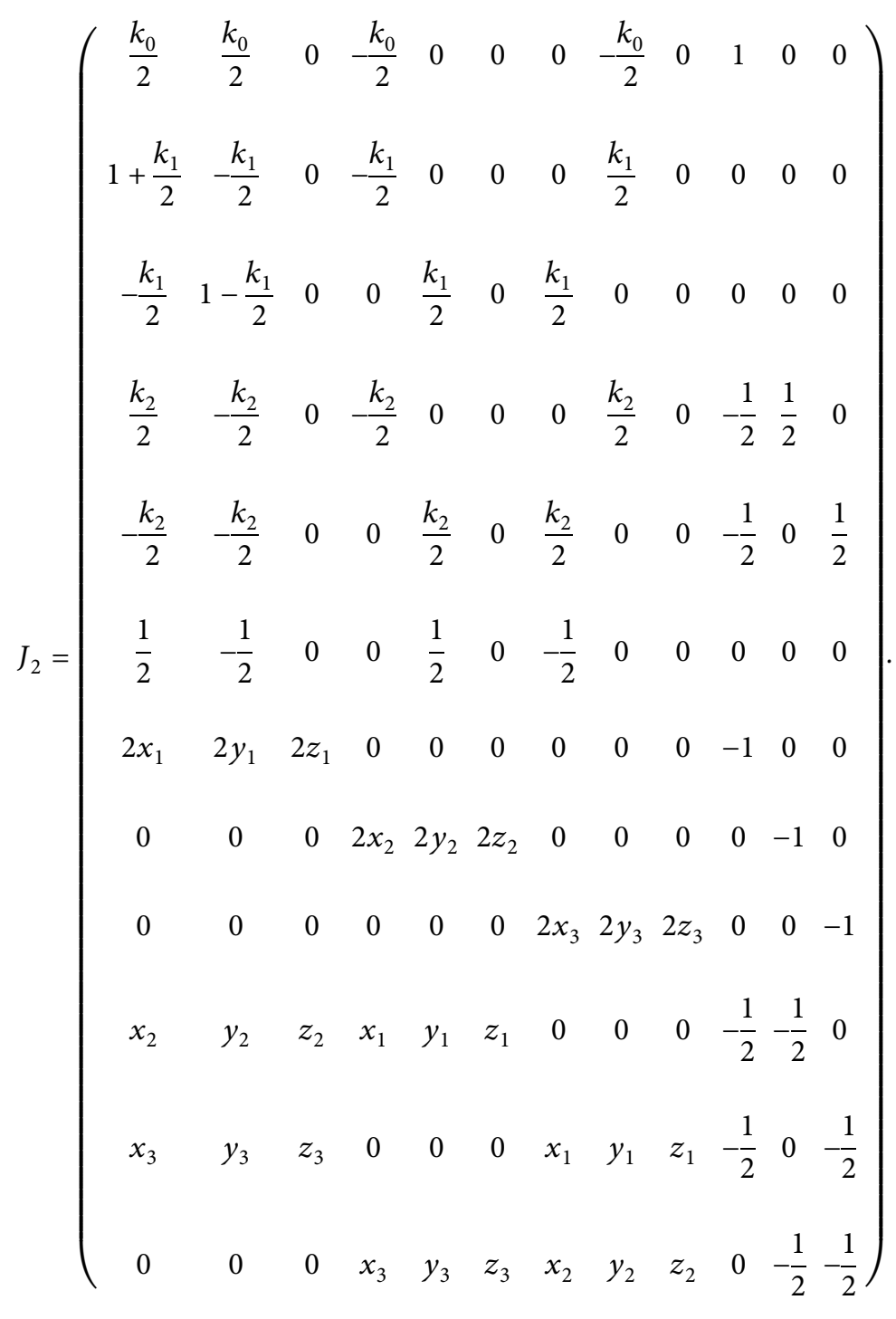


The six components $\left\{x_{1}, y_{1}, x_{2}, y_{2}, x_{3}, y_{3}\right\}$ represented with natural coordinates on the plane $\alpha \beta$ are solved out from the six linear equations in equation (22). Furthermore, $\left\{x_{1}, y_{1}, x_{2}, y_{2}, x_{3}, y_{3}\right\}$ are linear expressed with $\left\{\tau_{1}, \tau_{2}, \tau_{3}\right\}$ :

$$
\begin{aligned}
& x_{1}=-\frac{k_{1}+2 C_{4} k_{1}-2 C_{2} k_{2}+k_{1} \tau_{1}-k_{1} \tau_{2}}{2 k_{2}}, \\
& y_{1}=-\frac{k_{1}+2 C_{5} k_{1}-2 C_{3} k_{2}+k_{1} \tau_{1}-k_{1} \tau_{3}}{2 k_{2}}, \\
& x_{2}=-\frac{k_{0}+2 C_{4} k_{0}+2 C_{1} k_{2}+k_{0} \tau 1-2 k_{2} \tau 1-k_{0} \tau_{2}}{2 k_{0} k_{2}}-\frac{k_{1}+2 C_{4} k_{1}-2 C_{2} k_{2}+k_{1} \tau 1-k_{1} \tau_{2}}{2 k_{2}}, \\
& y_{2}=-\frac{-1-2 C_{5}-2 C_{6} k_{2}-\tau_{1}+\tau_{3}}{2 k_{2}}-\frac{k_{1}+2 C_{5} k_{1}-2 C_{3} k_{2}+k_{1} \tau_{1}-k_{1} \tau_{3}}{2 k_{2}}, \\
& x_{3}=-2 C_{6}-\frac{k_{1}+2 C_{4} k_{1}-2 C_{2} k_{2}+k_{1} \tau 1-k_{1} \tau_{2}}{2 k_{2}}-\frac{-1-2 C_{5}-2 C_{6} k_{2}-\tau_{1}+\tau_{3}}{2 k_{2}}, \\
& y_{3}=-\frac{C_{1}}{k_{0}}+\frac{1}{2 k_{2}}+\frac{C_{4}}{k_{2}}+\frac{\tau_{1}}{k_{0}}+\frac{\tau_{1}}{2 k_{2}}-\frac{\tau_{2}}{2 k_{2}}-\frac{k_{1}+2 C_{5} k_{1}-2 C_{3} k_{2}+k_{1} \tau_{1}-k_{1} \tau_{3}}{2 k_{2}} .
\end{aligned}
$$

After we substitute the expressions of $\left\{x_{1}, y_{1}, x_{2}, y_{2}, x_{3}, y_{3}\right\}$ in equation (26) into the Jacobian $J_{2}$ in equation (25), then $J_{2}$ becomes a new $12 \times 12$ dimensional expression with 6 variables $\left\{z_{1}, z_{2}, z_{3}, \tau_{1}, \tau_{2}, \tau_{3}\right\}$ and $6 \mathrm{DOF}$ parallel robot's structure parameters, $r_{a}, r_{b}, \theta_{a}, \theta_{b}$.

\subsection{Output Singularity Trajectory Equations Represented by} Natural Coordinates. $\tau_{1}, \tau_{2}, \tau_{3}$ are the coupling variables of position and orientation; therefore, the mobile platform is at singular configuration only when the determinant of Jacobian $J_{2}$ is zero while the singular configuration is expressed when the position and orientation are coupled. The singular trajectory equation of coupled pose is written as

$$
\operatorname{det}\left[J_{2}(\tau, z)\right]=0 \text {, }
$$

where remember $z=\left\{z_{1}, z_{2}, z_{3}\right\}^{T}$.

The specific form of Jacobian $J_{2}$ determinant after expansion is expressed as follows:

$$
\begin{aligned}
\operatorname{det}\left[J_{2}\right]= & s_{1} z_{1}^{3}+s_{2} z_{1}^{2} z_{2}+s_{3} z_{1} z_{2}^{2}+s_{4} z_{2}^{3}+s_{5} z_{1}^{2} z_{3}+s_{6} z_{1} z_{2} z_{3} \\
& +s_{7} z_{2}^{2} z_{3}+s_{8} z_{1} z_{3}^{2}+s_{9} z_{2} z_{3}^{2}+s_{10} z_{3}^{3},
\end{aligned}
$$

where $s_{i}$ is polynomial combinations of different degrees of $\tau_{1}, \tau_{2}, \tau_{3}$, namely, $s_{i}(\tau)$. Furthermore, $s_{i}$ is any combination polynomials with the highest degree to cubic orders of $\tau_{1}, \tau_{2}, \tau_{3}$.

If $\operatorname{det}\left[J_{2}\right]=0$ is true, then three major categories including seven secondary kinds of singular configurations are obtained from equation (28) as follows:

4.2.1. The First Category. $\operatorname{det}\left[J_{2}\right]=0$ when $z_{1}=z_{2}=z_{3}=0$ while the mobile platform and the base are coplanar.
However, this kind of output singularity is impossible if we consider the early stage of structural design.

\subsubsection{The Second Category}

(1) If $z_{1}=z_{2}=0$ and $s_{10}=0$, then $\operatorname{det}\left[J_{2}\right]=0$

(2) If $z_{2}=z_{3}=0$ and $s_{1}=0$, then $\operatorname{det}\left[J_{2}\right]=0$

(3) If $z_{1}=z_{3}=0$ and $s_{4}=0$, then $\operatorname{det}\left[J_{2}\right]=0$

In this case, there is a singular surface in $\tau_{1}, \tau_{2}, \tau_{3}$ space.

\subsubsection{The Third Category}

(1) If $z_{1}=0$ and $s_{4}=s_{7}=s_{9}=s_{10}=0$, then $\operatorname{det}\left[J_{2}\right]=0$

(2) If $z_{2}=0$ and $s_{1}=s_{5}=s_{8}=s_{10}=0$, then $\operatorname{det}\left[J_{2}\right]=0$

(3) If $z_{3}=0$ and $s_{1}=s_{2}=s_{3}=s_{4}=0$, then $\operatorname{det}\left[J_{2}\right]=0$

In this case, in $\tau_{1}, \tau_{2}, \tau_{3}$ space, the intersections of four curved surfaces are singular points, lines, and surfaces.

\section{Example}

5.1. Preliminary Definitions. Take the planar platform 6-UPS parallel robot for example, as shown in Figure 2, where the geometric structure parameters are $\theta_{a}=0.28618 \pi \mathrm{rad}$, $\theta_{b}=0.046988 \pi \mathrm{rad}, r_{a}=0.849864 \mathrm{~m}, r_{b}=0.849864 \mathrm{~m}$. The six pairs of vertices $P_{a}$ of the mobile and base platforms are circularly and symmetrically arranged on a plane circle, as shown in Figure 3.

5.2. Output Singularities. Substituting the structural parameters into $\operatorname{det}\left[J_{2}\right]$, the numerical expression of the singular trajectory is obtained as follows:

$$
\operatorname{det}\left[J_{2}\right]=f\left(z_{1}, z_{2}, z_{3}, \tau_{1}, \tau_{2}, \tau_{3}\right) .
$$




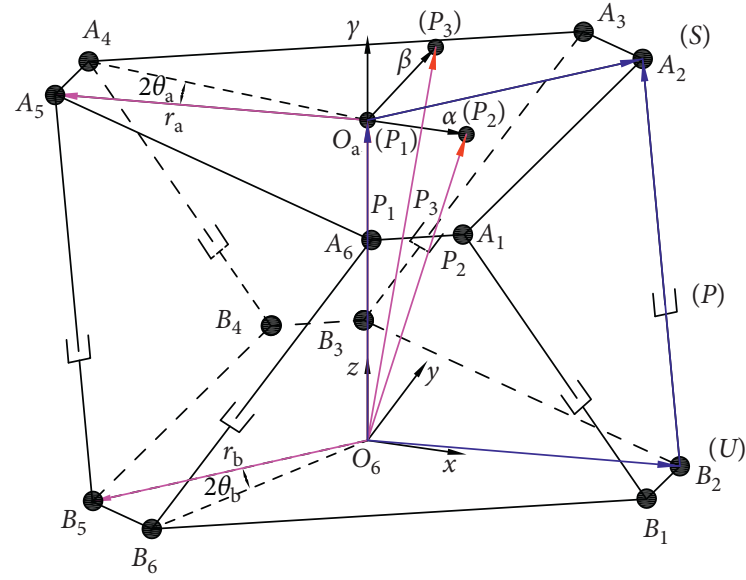

FIGURE 2: Diagram of 6-UPS planar parallel robot. There are two platforms and six legs. The passive joints $A_{i}$ connecting the moving platform are spherical hinge $S$. The passive joints $B_{i}$ connecting the base are universal joint $U$. The active joints $P i$ driving the legs are prismatic joint $\mathrm{P}$ fixed on each leg. $O_{a} \alpha \beta \gamma$ is the relative moving frame. $O_{b} x y z$ is the absolute static frame. $P_{1}$, $P_{2}, P_{3}$ are selected as natural coordinates. $O_{a}$ and $O_{b}$ are the circle center of the two platforms. $r_{a}, r_{b}$ are circumradii of the base and the moving platform, respectively. $\theta_{a}, \theta_{b}$ are center semiangles corresponding to the short side of the platform, respectively.

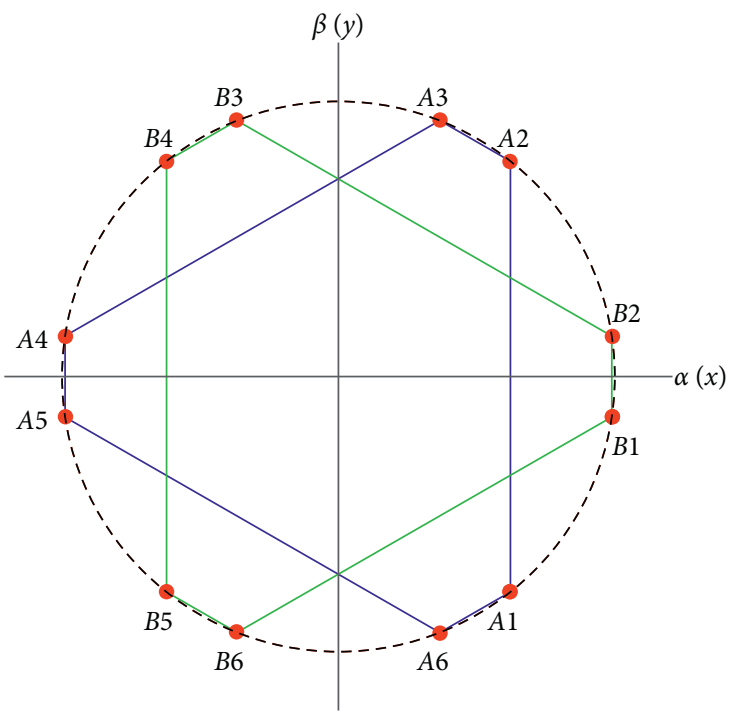

FIGURE 3: Diagram of vertices arrangement for 6-UPS planar parallel robot. $A_{i}$ are the vertices of the moving platform. $B_{i}$ are the vertices of the base. The circles in the dashed line are the circumscribed circle of the moving and base platforms, respectively.

In the following, we study the output singular types of the mechanism and the spatial distribution of singular trajectories. If $\operatorname{det}\left[J_{2}\right]=0$ is true, three kinds of output singularities obtained in Section 4.2 are analyzed as follows.

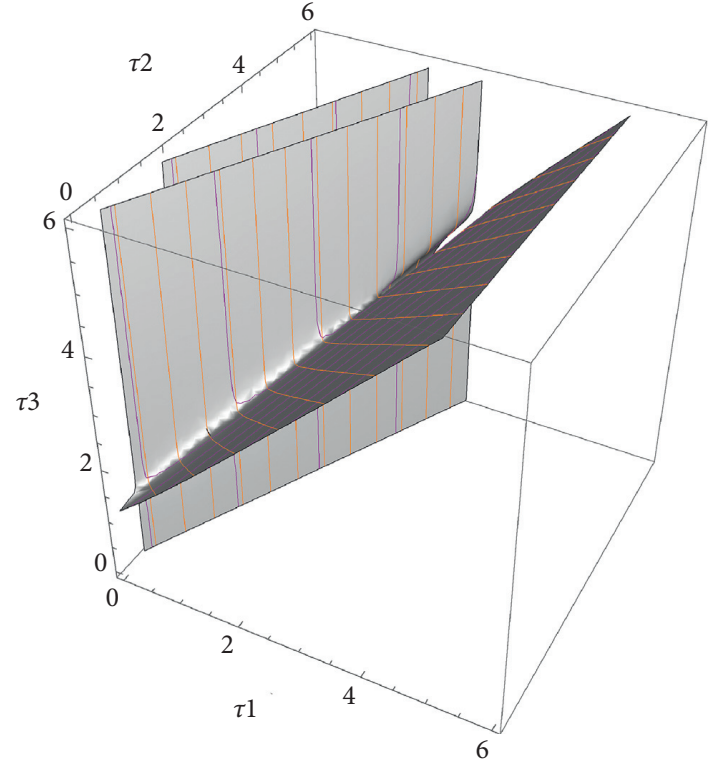

Figure 4: Case 1 of class II output singular trajectories. $\tau_{1}, \tau_{2}, \tau_{3}$ are the coupling variables of position and orientation.

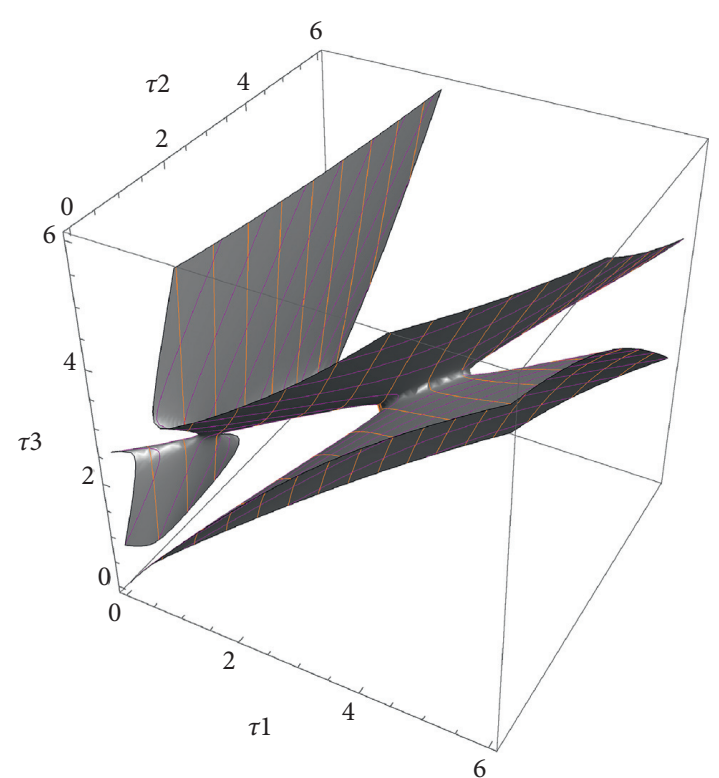

FIgURE 5: Case 2 of class II output singular trajectories. $\tau_{1}, \tau_{2}, \tau_{3}$ are the coupling variables of position and orientation.

5.2.1. The First Category. It is obviously impossible. .

\subsubsection{The Second Category}

(1) If $z_{1}=z_{2}=0$ and $s_{10}=0$, then $\operatorname{det}\left[J_{2}\right]=0$. The spatial distribution of singular trajectories is shown in Figure 4.

(2) If $z_{2}=z_{3}=0$ and $s_{1}=0$, then $\operatorname{det}\left[J_{2}\right]=0$. The spatial distribution of singular trajectories is shown in Figure 5. 


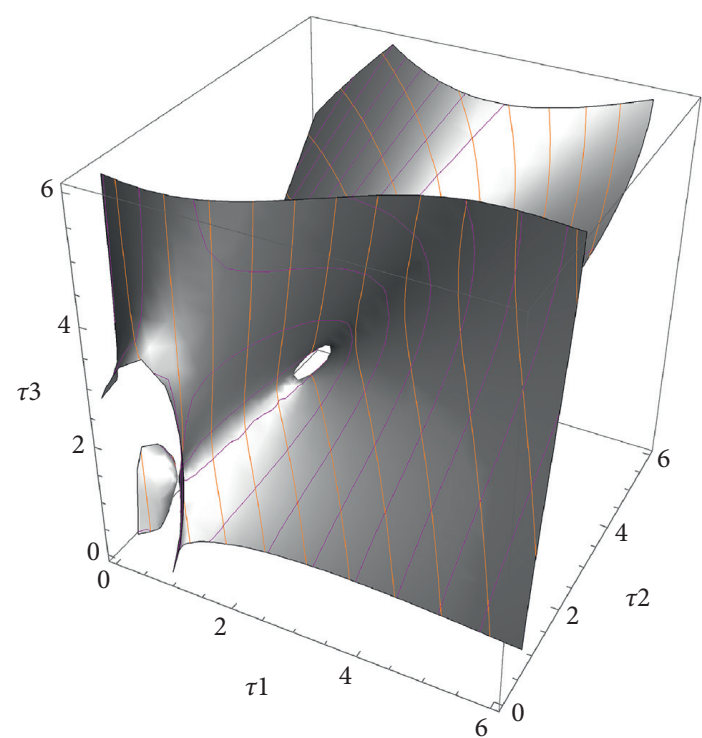

FIgURE 6: Case 3 of class II output singular trajectories. $\tau_{1}, \tau_{2}, \tau_{3}$ are the coupling variables of position and orientation.

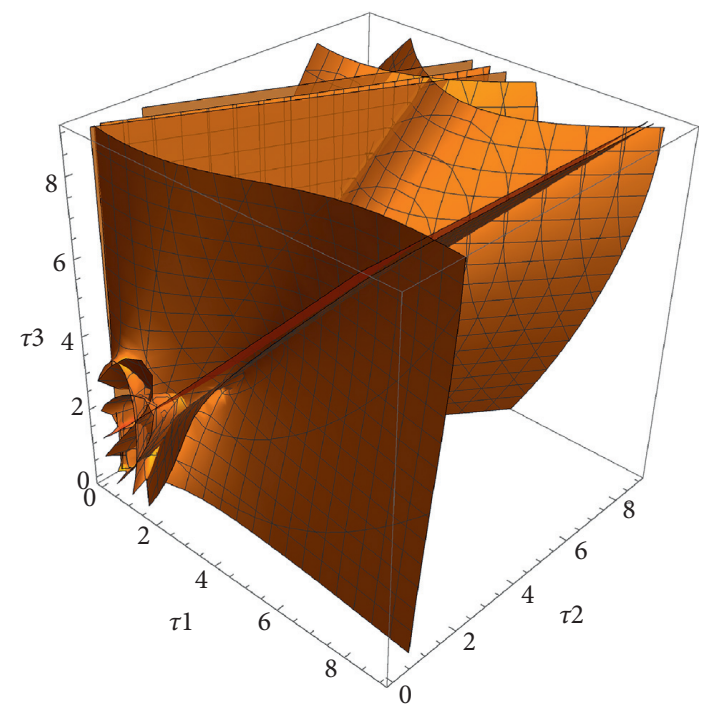

FIgURE 7: Case 1 of class III output singular trajectories. $\tau_{1}, \tau_{2}, \tau_{3}$ are the coupling variables of position and orientation.

(3) If $z_{1}=z_{3}=0$ and $s_{4}=0$, then $\operatorname{det}\left[J_{2}\right]=0$. The spatial distribution of singular trajectories is shown in Figure 6.

According to the 6-UPS structure, we know that $z_{1} \neq 0$. As a result, the two cases, (1) and (3), are impossible. Therefore, only case (2) exists in this kind of output singularities. What is more, there is a three-dimensional curved surface, $s_{1}=0$, of singular trajectory with six-dimensional parameters for coupled position and orientation in space $\tau_{1}, \tau_{2}, \tau_{3}$.

\subsubsection{The Third Category}

(1) If $z_{1}=0$ and $s_{4}=s_{7}=s_{9}=s_{10}=0$, then $\operatorname{det}\left[J_{2}\right]=0$. The spatial distribution of singular trajectories is shown in Figure 7.

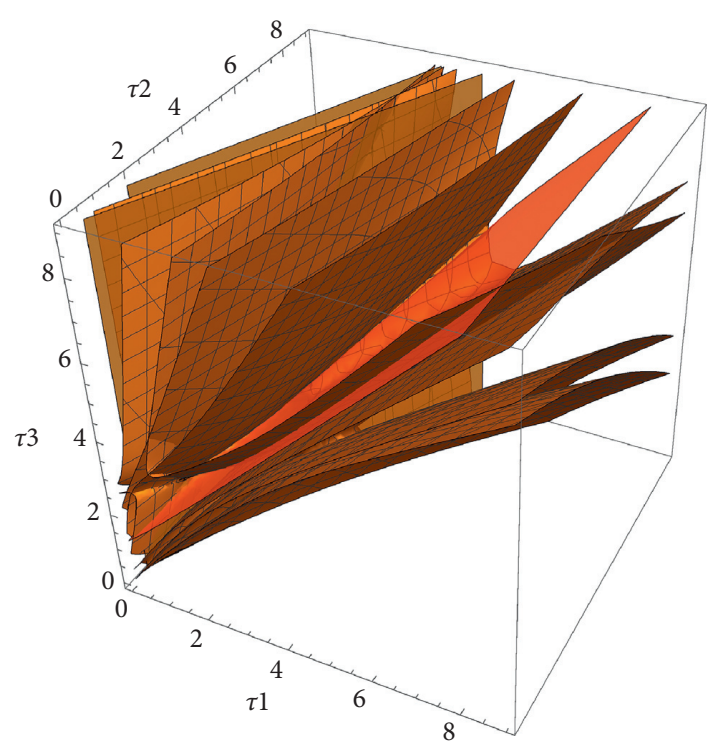

FIgURE 8: Case 2 of class III output singular trajectories. $\tau_{1}, \tau_{2}, \tau_{3}$ are the coupling variables of position and orientation.

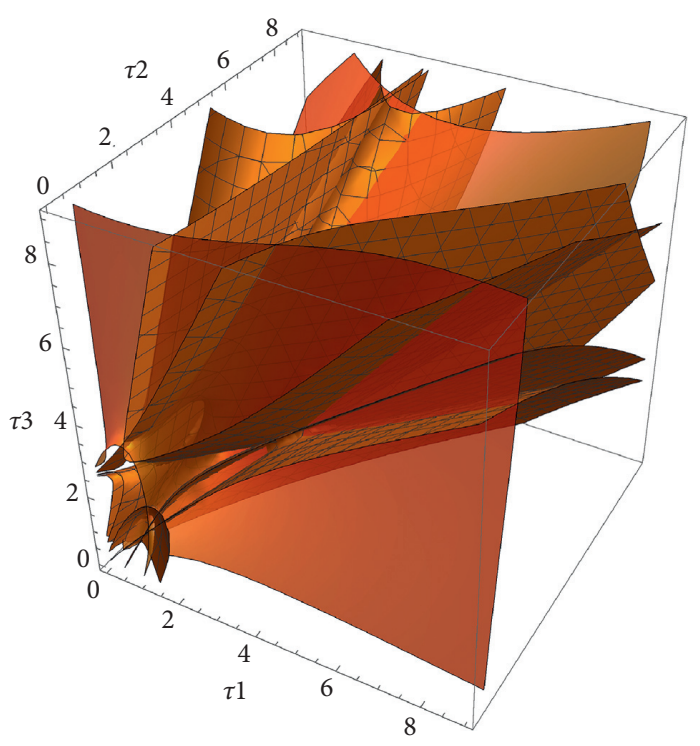

Figure 9: Case 3 of class III output singular trajectories. $\tau_{1}, \tau_{2}, \tau_{3}$ are the coupling variables of position and orientation.

(2) If $z_{2}=0$ and $s_{1}=s_{5}=s_{8}=s_{10}=0$, then $\operatorname{det}\left[J_{2}\right]=0$. The spatial distribution of singular trajectories is shown in Figure 8.

(3) If $z_{3}=0$ and $s_{1}=s_{2}=s_{3}=s_{4}=0$, then $\operatorname{det}\left[J_{2}\right]=0$. The spatial distribution of singular trajectories is shown in Figure 9.

According to the 6-UPS structure, we know that $z_{1} \neq 0$. As a result, case (1) is impossible. Therefore, only cases (2) and (3) exist in this kind of output singularities. What is more, there are three-dimensional singular trajectories of points, lines, and surfaces, which are the intersections of the four curved surfaces, $s_{1}=s_{5}=s_{8}=s_{10}=0$ and $s_{1}=s_{2}=s_{3}=s_{4}=0$, respectively, 
with six-dimensional parameters for coupled position and orientation in space $\tau_{1}, \tau_{2}, \tau_{3}$.

\section{Conclusions and Future Work}

The natural coordinates method is used to represent position $P$ and orientation $R$. Then, a set of kinematic models and two sets of constraint models are represented as quadratic equations. These models contain quadratic terms, first-order terms, and constant terms, in which the highest order term is quadratic. Thus, the simplest and nonlinear kinematic models and constraint models for a kind of planar platform type $6 \mathrm{DOF}$ parallel robot are established. The Jacobian matrix only contains the first-order term and constant term after derivation; namely, the highest order is the first order. Furthermore, position $P$ and orientation $R$ are the unified representation of natural coordinates. Therefore, there is no need for dimensionless processing. What is more, it provides a basic guarantee for the optimization of analytical expressions of singular trajectories.

The kinematic models and constraint models of the planar platform type 6DOF parallel robot are successfully combined and the analytical expressions of output singularities are obtained by using the natural coordinates method. Then, the types of output singularities are discussed and the distributions of singular trajectories in space are studied too. For the output singularities, the sixdimensional coupled variables of position $P$ and orientation $R$ are described in three dimensions for the first time. Namely, the six-dimensional singular trajectory points, lines, and surfaces are represented with six-dimensional coupled variables of the position $P$ and orientation $R$ in $3 \mathrm{D}$ space. The proposed method realizes the $3 \mathrm{D}$ full visualization of six-dimensional variables and solves the problem of solution of orientation singularity fixing the position $P$ or solution of position singularity fixing the orientation $R$. In the end, the singular trajectory points, lines, and surfaces of output singularities are fully drawn by a numerical example.

Further research shows that the singularities research based on the natural coordinates method lays a solid foundation for solving the $6 \mathrm{D}$ free singularity workspace of planar platform type 6DOF parallel robot based on the forward kinematics solution and realizing $3 \mathrm{D}$ complete visualization description. What is more, the results of the singularities research can be applied to increase the precision of machine-building parts [20]. Due to the limitation of an article-length, the related results on the free singularity workspace will be published in another paper.

\section{Data Availability}

The data used to support the findings of this study are available from the author upon request.

\section{Conflicts of Interest}

The authors declare that they have no conflicts of interest.

\section{Acknowledgments}

The authors disclosed receipt of the following financial support for the research, authorship, and/or publication of this article. This research was supported by the National Natural Science Foundation of China (Grant no. 51975277). The authors gratefully acknowledge these support agencies.

\section{References}

[1] K. H. Hunt, Kinematic Geometry of Mechanisms, Claredon Press, Oxford, UK, 1978.

[2] L. Yan-Wen, Z. Huang, and F. Gao, "A new method of singularity research and its example in application," Journal of Yanshan University, vol. 28, no. 1, pp. 40-48, 2004.

[3] Y. Cao, C. M. Gosselin, H. Zhou et al., "Orientation-singularity analysis and orientationability evaluation of a special class of the Stewart Gough parallel manipulators," Robotica, vol. 31, no. 8, pp. 1361-1372, 2013.

[4] P. Laryushkin, V. Glazunov, and K. Erastova, "On the maximization of joint velocities and generalized reactions in the workspace and singularity analysis of parallel mechanisms," Robotica, vol. 37, no. 4, pp. 675-690, 2019.

[5] C. Gosselin and J. Angeles, "Singularity analysis of closed-loop kinematic chains," IEEE Transactions on Robotics and Automation, vol. 6, no. 3, pp. 281-290, 1990.

[6] K. Y. Tsai, J. C. Lin, and Y. Lo, "Six-DOF parallel manipulators with maximal singularity-free joint space or workspace," Robotica, vol. 32, no. 3, pp. 401-411, 2014.

[7] X. Yang, H. Wu, B. Chen, Y. Li, and S. Jiang, "A dual quaternion approach to efficient determination of the maximal singularityfree joint space and workspace of six-DOF parallel robots," Mechanism and Machine Theory, vol. 129, pp. 279-292, 2018.

[8] K. H. Hunt, "Structural kinematics of in-parallel-actuated robot-arms," Journal of Mechanisms, Transmissions, and Automation in Design, vol. 105, no. 4, pp. 705-712, 1983.

[9] J.-P. Merlet, "Singular configurations of parallel manipulators and Grassmann geometry," The International Journal of Robotics Research, vol. 8, no. 5, pp. 45-56, 1989.

[10] K. Wen, T. Seo, and J. W. Lee, “A geometric approach for singularity analysis of 3-DOF planar parallel manipulators using Grassmann-Cayley algebra," Robotica, vol. 35, no. 3, pp. 511-520, 2017.

[11] J. Ma, Q. Chen, H. Yao, X. Chai, and Q. Li, "Singularity analysis of the 3/6 Stewart parallel manipulator using geometric algebra," Mathematical Methods in the Applied Sciences, vol. 41, no. 6, pp. 2494-2506, 2018.

[12] H.-B. Choi, A. Konno, and M. Uchiyama, "Analytic singularity analysis of a 4-DOF parallel robot based on Jacobian deficiencies," International Journal of Control, Automation and Systems, vol. 8, no. 2, pp. 378-384, 2010.

[13] H.-B. Choi and J. Ryu, "Singularity analysis of a four degreeof-freedom parallel manipulator based on an expanded $6 \times 6$ Jacobian matrix," Mechanism and Machine Theory, vol. 57, pp. 51-61, 2012.

[14] M. Ceccarelli, P. Maurizio Decio Fino, and J. Manuel Jimenez, "Dynamic performance of CaPaMan by numerical simulations," Mechanism and Machine Theory, vol. 37, no. 3, pp. 241-266, 2002.

[15] B. Li, Y. Cao, Q. Zhang, and Z. Huang, "Position-singularity analysis of a special class of the Stewart parallel mechanisms with two dissimilar semi-symmetrical hexagons," Robotica, vol. 31, no. 1, pp. 123-136, 2013. 
[16] B. M. St-Onge and C. M. Gosselin, "Singularity analysis and representation of the general Gough-Stewart platform," The International Journal of Robotics Research, vol. 19, no. 3, pp. 271-288, 2000.

[17] B. Li, Y. Cao, Q. Zhang, and C. Wang, "Singularity representation and workspace determination of a special class of the Gough-Stewart platforms," International Journal of Advanced Robotic Systems, vol. 10, no. 11, p. 378, 2013.

[18] A. Kilin, P. Bozek, Y. Karavaev, A. Klekovkin, and V. Shestakov, "Experimental investigations of a highly maneuverable mobile omniwheel robot," International Journal of Advanced Robotic Systems, vol. 14, no. 6, Article ID 172988141774457, 2017.

[19] R. Pirník, M. Hruboš, D. Nemec, T. Mravec, and P. Božek, Integration of Inertial Sensor Data into Control of the Mobile Platform, Springer International Publishing, New York, NY, USA, 2015.

[20] P. BoEk, A. Lozkin, and A. Gorbushin, "Geometrical method for increasing precision of machine building parts," Procedia Engineering, vol. 149, pp. 576-580, 2016. 